\title{
Single Image Haze Removal Using Dark Channel Prior and Adaptive Transmission Rate
}

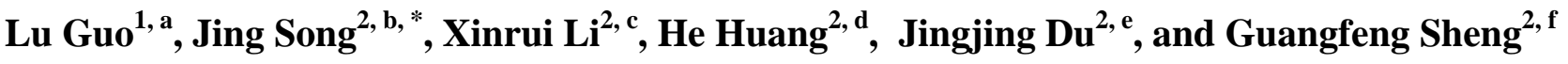 \\ ${ }^{1} \mathrm{Xi}$ ' an ASN Technology Group Company, Xi' an 710065, China \\ ${ }^{2}$ School of Electronic and Control Engineering, Chang' an University, Xi' an, 710064, China \\ a tolovelulu@126.com, ${ }^{b} 1158940754 @ q q . c o m,{ }^{c} 1076359350 @ q q . c o m,{ }^{d}$ huanghe@chd.edu.cn \\ 5597248333@qq.com, ${ }^{e}$ 1362429596@qq.com.
}

Keywords: haze removal, dark channel, image processing, computer simulation, algorithm

\begin{abstract}
In order to solve the problems of low clarity and brightness, color distortion of the restored image obtained by the traditional haze removal algorithm, this paper proposes a new single image haze removal algorithm using dark channel prior and adaptive transmittance rate. Firstly, the image is partitioned into several local regions to calculate the dark channel and minimum map of haze image. Secondly, the atmospheric light estimation is calculated according to the dark channel map, and the atmospheric dissipation value of each pixel is calculated by the atmospheric dissipation function. Then, combined with the atmospheric dissipation function, the median guide filtering algorithm is used to distinguish the image into the close range and a remote area, and the atmospheric dissipation function value of the close range is corrected to get the accurate estimation of the transmittance. Finally, the restoration image is obtained based on the atmospheric scattering model. The experimental results show that compared with the traditional algorithm, the fuzzy coefficient of the image is reduced by $23.09 \%$, the structure similarity and the tone reduction degree are increased by $14.63 \%$ and $12.29 \%$ respectively, and the quality of the restored image can be significantly improved.
\end{abstract}

\section{Introduction}

In hazy weather, severe attenuation occurs when the incident light reaches the image acquisition device through the air, resulting in blurred images and low contrast and color saturation. This is because the particles, such as water droplets and dust suspended in the air, on the light scattering and refraction, so that light outside part of the target scene may be scattered and refracted multiple times into the photosensitive element, and part of the light in the target scene is due to The scattering has been severely attenuated, causing a certain change in the intensity and wavelength of the light, resulting in severe distortion of the image acquired in the haze.

At present, the methods of image defogging can be roughly divided into two categories: image enhancement-based fogging and image removal based on physical models. The disadvantage of the 
former is that it does not consider the factors that cause the image's degradation, and the fog is removed only by enhancing the image's contrast. Therefore, the fog is essentially not removed, and the effect is not ideal [1]. In a study of image-removal methods based on physical models, Tan K K et al proposed the statistical degradation model [2] and applied it to the visibility restoration of colour fog images. These methods are based on the scene depth of an image being known; in general, the depth information of the fog image being detailed depends on using high-precision distance-measuring equipment. Therefore, these methods have some limitations in practical application.

In recent years, many researchers have turned to single image defogging methods based on prior knowledge or hypotheses [3]. He et al [4] found, using statistics from a large number of images, that for a clear image area that does not contain the sky, when taking the minimum value of the pixels $R, G$, and $\mathrm{B}$, the value that always tend toward 0 is defined as a dark channel. The dark channel is used as a priori information to estimate the atmospheric light and transmittance. At the same time, a rough estimate of the transmittance is obtained through a soft ridge map and filtered to obtain the accurate transmittance, so that a clear image can be recovered by using an imaging model under hazy weather. Although soft matting can achieve good transmittance estimation, the amount of calculation is too large, since then, many scholars have proposed a series of improved algorithms, such as Wang et al proposed the bilateral filtering dehazing algorithm [5], He et al proposed the guided filtering defogging algorithm [6], but this kind of algorithm only changes the filter. Because of the defects of the transmission estimation algorithm, the restored image is dim, the color distortion is easy and the image quality is not high.

Based on the atmospheric scattering model, this paper analyzes the degradation process of the image under the haze weather. In view of the problem that the traditional image defog algorithm is inaccurate in the inaccurate estimation of the transmittance, the image brightness is low and the color distortion is distorted. A single image haze removal algorithm based on the dark channel theory and the transmission adaptive is proposed.

\section{Model of Image Degradation in Hazy Weather}

In hazy weather, airborne particles in the air scatter and refract light, which can make the image blurred and decrease the quality of the captured image. In the common dehazing algorithm, when analyzing images taken in hazy weather, one must take into account the influence of the media on the imaging - that is to say, the incident light attenuation and atmospheric optical imaging process — and then build the model to analyse image quality decline under fog and haze conditions. We established such a model by analyzing the effects of fog and haze on a camera's imaging, as shown in Figure 1[7]. We can determine the influence of fog and haze particles on light and imaging by analysing the model.

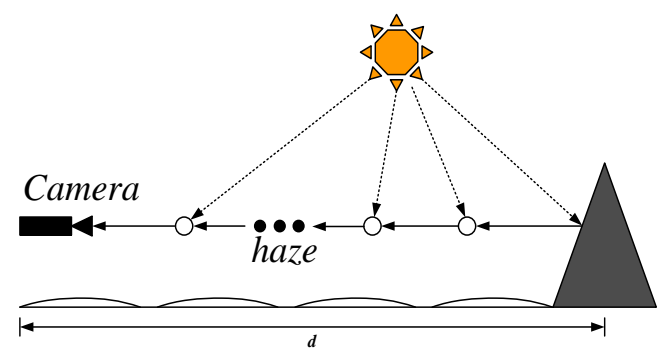

Fig. 1 Effect of fog and haze on image 


$$
L(x, y)=L_{0}(x, y) e^{-k d(x, y)}+L_{s}\left(1-e^{-k d(x, y)}\right)
$$

Formula 1 is composed of an incident light attenuation model and an atmospheric optical-imaging model, in which $L(x, y)$ is a fog-degraded image captured by a visible lens, $L_{0}(x, y) e^{-k d(x, y)}$ is the attenuation model of incident light, and $L_{s}\left(1-e^{-k d(x, y)}\right)$ is the atmospheric optical-imaging model. Due to the scattering effect of atmospheric particles, part of the incident light cannot reach the lens, causing the incident light to decay. In the incident light attenuation model, $L O(x, y)$ represents the intensity of the reflected light at that point, namely the inherent intensity which is not attenuated. The attenuation rate of the incident light is related to the distance from the scene to the receiving device. $d(x, y)$ is the scene depth, and $k$ is the atmospheric scattering coefficient.

In the atmospheric optical imaging model, $L_{s}$ is ambient luminance, and $e^{-k d(x, y)}$ is the transmittance distribution. On the basis of the model analysis, images degrade in hazy weather because the haze particles $(1-10 \mu \mathrm{m})$ are far larger than the size of pure air particles (about 10-4 $\mu \mathrm{m}$ ), the particle size determines the scattering coefficient. At this point, the visible light scattering coefficients of different colors can be considered similar; that is, there is an equal scattering of all visible light. Therefore, the images obtained in hazy weather is gray and unclear. In addition, due to the influence of haze particles on light scattering, the incident light attenuation model $L_{0}(x, y) e^{-k d(x, y)}$ shows that the intrinsic brightness of the image is exponentially attenuated, influenced by the depth, which also decreases the fog image's brightness, and the haze particles will also produce more noise on the light scattering and refraction, resulting in poor image quality.

\section{Dark Channel Prior Theory}

Through the statistics of a large number of fog free images and analysis of the statistical law, He proposed the dark channel prior theory[4], that is, the fact that there is a dark channel in the image: a fog free clear image is divided into a number of local regions, in most local regions there is a rule that the value of some pixels in a color channel in RGB is close to 0 in most local regions. The formula is expressed as follows:

$$
J^{\text {dark }}(x)=\min _{y \in \Omega(x)}\left(\min _{c \in\{r, g, b\}} J^{c}(y)\right)
$$

In the formula, $\Omega(x)$ represents a local area with pixel $x$ as the center, $y$ is the adjacent pixel in the region, and $J_{c}(y)$ is the value of a colour channel of its corresponding $R, G, B . J_{\text {dark }}(x)$ is a dark channel value corresponding to the pixel point $x$.

In the scene restoration method based on the physical model, in order to easily obtain the restored image, it is necessary to perform equivalent transformation on the formula (1) such that $I(x)=L(x)$, $J(x)=L_{0}(x), t(x)=e^{-k d(x)}, A=L_{s}$, a simplified image degradation model under haze weather can be obtained as follows:

$$
I(x)=J(x) t(x)+A(1-t(x))
$$

In the formula, $J(x)$ represents the restoration image, and $t(x)$ is called the transmission rate which reflecting the ability of ray to penetrate haze. It contains depth of field information from 0 to $1, I(x)$ represents foggy images, and A represents atmospheric intensity, that is, the intensity of light at infinity.

The formula (3) is slightly deformed, and the results are as follows:

$$
\frac{I^{c}(x)}{A^{c}}=t(x) \frac{J^{c}(x)}{A^{c}}+1-t(x) \quad c \in\{r, g, b\}
$$


In practice, since the pixels in the image have correlation, in order to facilitate the calculation, when estimating the transmittance, it is assumed that the transmittance is the same in the local small range. So, according to the dark channel prior theory, the rough estimate of transmittance can be obtained.

$$
t(x)=1-w \min _{c}\left\{\min _{y \in \Omega(x)}\left[\frac{I^{c}(y)}{A^{c}}\right]\right\}
$$

In reality, even on a sunny day, the image acquired by the acquisition device will contain a small amount of fog. Therefore, in order to be closer to reality, it is necessary to add a coefficient $w$ to remove a certain degree of fog when defogging, in this article, the value of $w$ is 0.95 .

\section{Design of Image Haze Removal Algorithm Based on Adaptive Transmittance}

\subsection{Traditional atmospheric light solution method and impact analysis}

The image dehazing algorithm based on the foggy image degrading model focuses on estimating depth of field information or transmittance. The more common transmission estimation method is to use normalized dark channel image as the depth of field information, and to use it as the transmittance after further processing, as shown in formula (5). However, this method has great limitations.

A large number of studies have shown that in some special cases, the restoration images obtained by the traditional dark channel priori dehazing algorithm are prone to distortion in bright areas, especially in the near field. The pixels in these areas are generally high in brightness and tend to be white, such as sky, white buildings, strong light, etc. According to formula (5), since the pixel value of the bright region is high, the minimum value of the local area in the image does not tend to 0 , the dark channel prior theory is not established at this time. However, the value of $I_{c}(y)$ will be very close to the $A_{c}$ in formula (5). The calculated transmittance of the white object and the bright part is approaching and zero. Defogging algorithm based on dark priori is invalid in these areas, no matter how to adjust the parameters (filter window size, etc.), it can not solve this problem. Obviously, there is a big deviation between the estimated transmittance and the reality. As a result, the brightness and color saturation of the local area are reduced after the fog is removed, and the reconstructed image is distorted.

\subsection{Solving method of adaptive transmittance}

From the previous analysis, it can be seen that the dark channel prior theory is not valid in the bright region. If these bright areas exist in the near view part of the image, serious distortion will occur in the restored image. Therefore, this paper proposes an adaptive method to solve the transmittance and applies it to the image defogging process. By constructing an atmospheric dissipation function, it can estimate and modify the transmittance adaptively, so as to solve the problem of failure of dark channel priori theory in bright region.

First, through the atmospheric scattering model introduced above, the atmospheric dissipation function is defined as follows, where $\mathrm{A}$ is the atmospheric light estimation, $t(x)$ is the transmittance estimation, and $V$ is the atmospheric dissipation value:

$$
V=A(1-t(x))
$$

For the above deformation, the transmittance can be estimated as: 


$$
t(x)=1-\frac{V}{A}
$$

According to the prior theory of dark channel, the regions with high fog concentration in the image are relatively bright in the dark channel image, and the fog concentration corresponds to the degree of light dissipation.Therefore, it can be considered that the brightness of pixel points in the dark channel image represents the dissipation degree of light through the atmosphere. In order to simplify the calculation, the minimum channel graph is adopted to replace the dark channel graph, that is, the value of the atmospheric dissipation function $\mathrm{V}$ is equivalent to:

$$
V=I_{\min }=\min _{c \in\{r, g, b\}}\left(I^{c}\right)
$$

Obviously, the minimum channel graph contains a lot of details such as the edge of the original foggy image, and it is not practical to estimate the scene depth directly. On the other hand, in general, the concentration of fog in the near view area is small and contains more high-frequency details such as edges. The intensity of the edge details in the minimum channel graph is related to the scene depth. Therefore, the intensity of the detail information in the image can be estimated from the adaptive adjusted transmittance according to the intensity of the detail information in the image

Meter. In this paper, the improved median guide filtering is used to filter the image, and the difference image before and after filtering is taken as the high frequency information in the image, the corresponding close-up part of the image, and the atmospheric dissipation function of the close-up part is modified.

Guide Filter is a kind of fast Filter with excellent performance. It can not only maintain the characteristics of the Guide image in the output image, but also have a fairly good filtering effect on the filtered image.In this paper, on the basis of the guide filter, a kind of Median-Guide Filter with simplified calculation is designed, whose formula is as follows:

$$
\begin{gathered}
q_{i}=a_{k} I_{i}+b_{k}, \forall i \in \omega_{k} \\
a_{k}=\frac{\left(I_{k}^{m}-p_{k}^{m}\right)^{2}}{\varepsilon+\left(I_{k}^{m}\right)^{2}-\left(p_{k}^{m}\right)^{2}} \\
b_{k}=p_{k}^{m}-a_{k} \cdot p_{k}^{m}
\end{gathered}
$$

Among them, the $q_{i}$ is the Median-Guide Filter filtering output, Ii is the input boot image, $a_{k}$ and $b_{k}$ are respectively filter coefficients, and the value is defined as a constant in the local region. $I_{k}{ }^{m}$ represents the boot figure I in the local window $w_{k}$ the median value of the inside pixel, $p_{k}{ }^{m}$ is the median value of the image $p$ to be filtered in the window corresponding to the position in the boot image. In this paper, the gray scale image of foggy image is used as the guide image, and the minimum channel image I is used $I{ }_{\min }$ As the image to be filtered, the rough estimated transmittance $V^{\prime}$ is obtained by filtering it, and its formula is as follows:

$$
V^{\prime}=\operatorname{Guide}\left(\operatorname{rgb} 2 \operatorname{gray}(I), I_{\min }\right)
$$

According to the previous analysis, if the original image is bright in the close-up area, the pixel points obtained in the smallest channel map will also be bright, and the corresponding scattering function value will be large, which will misunderstand the close-up image as the perspective area with relatively dense fog and relatively large depth of field, which is obviously not in line with the reality.Therefore, it is necessary to modify the atmospheric dissipation function of gas rough 
estimation, which is defined as:

$$
\Delta c=\left|V^{\prime}-I_{\min }\right|
$$

$\Delta c$ Represents the high frequency texture details separated from the minimum channel graph by the median guide filter.Because the texture information of the close-range area is obvious, the corresponding value is large. $\Delta c$ The modified atmospheric scattering function is defined as:

$$
V^{\prime \prime}=V^{\prime}\left(1-\omega^{*} \Delta c\right)
$$

It can be seen from formula (14) that the scattering function value of the close-range area is weakened to a certain extent, that is, the light scattering in the close-range area is not large, so the resulting scattering function value is more practical and accurate.

\section{Experimental Results and Analysis}

The hardware platform of this experiment is Inter(R) Core (TM) i5-3230m CPU@2.6GHz, 4GB RAM, software platform is Matlab2017a, and windows7 professional. In the experiment, 300 images containing haze were selected for testing. Here, four groups of images were randomly selected. The dehazing method of He et al [4] and Wang. et al are selected as the contrast algorithm. The experimental results are shown in figure 2.

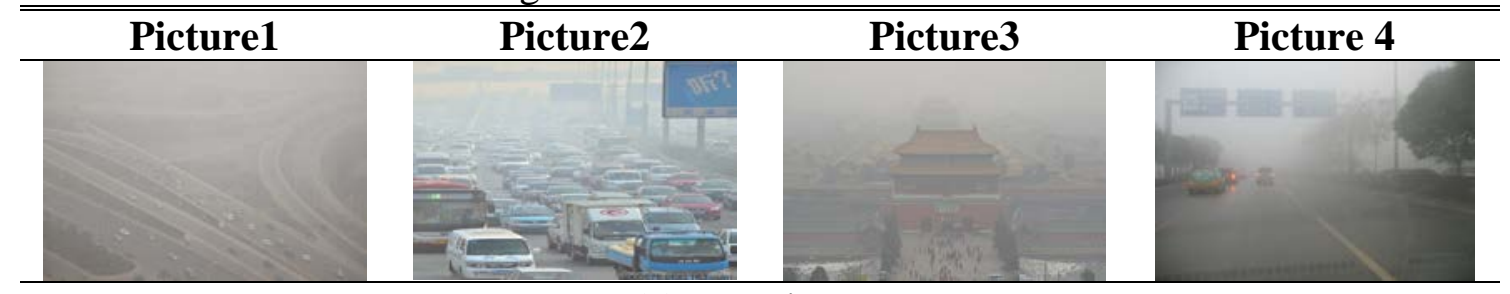

(a) Haze image

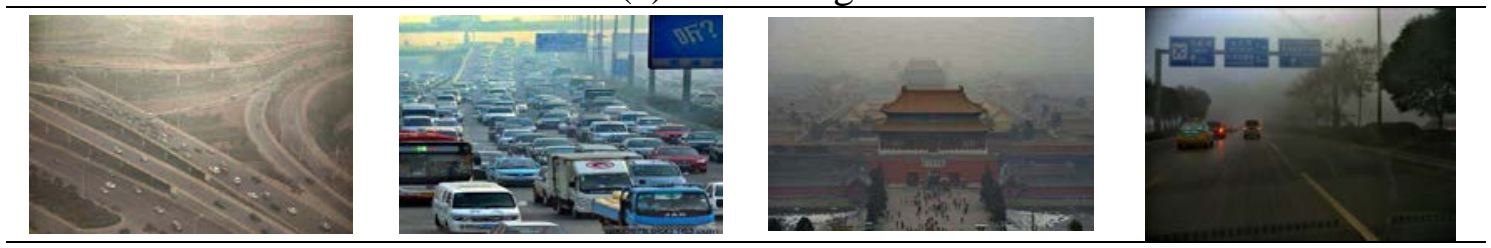

(b) He. et al

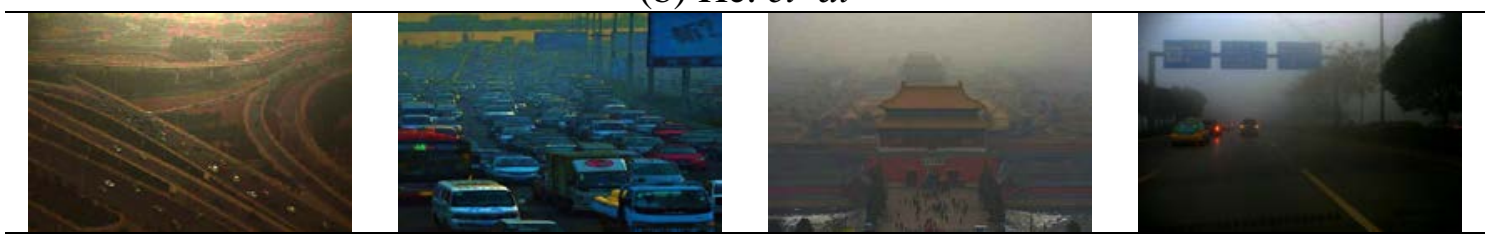

(c) Wang. et al

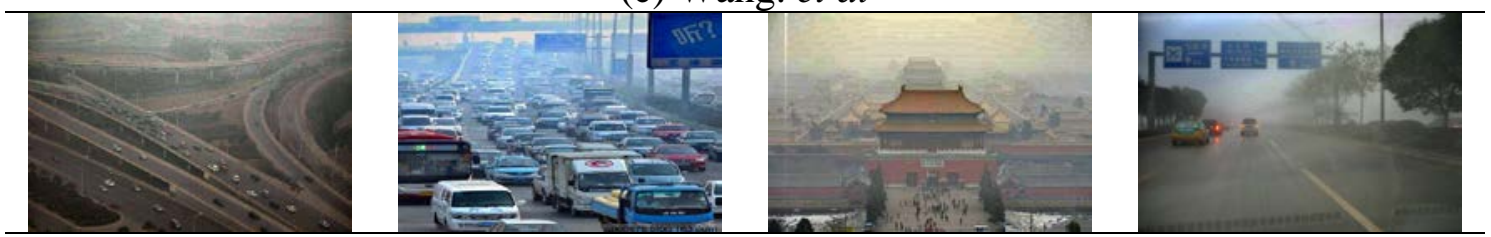

(e) our method

Fig. 2 comparison of algorithm

In figure 2, (a) is the four randomly selected images containing fog, (b), (c) and (d) are the recovered images obtained by applying different algorithm. It can be seen from the subjective visual effect that the restoration image obtained by the algorithm in this paper is clearer, the brightness and 
color saturation are higher, and the tone is more consistent with the original scene. Table 1 shows the objective evaluation index data of the restored image [8], where, fuzzy coefficient (Kblur), structural similarity (SSIM) and tonal reduction are selected for comparison. As the definition of Kblur, the smaller the value, the clearer the image will be, and the higher the structure similarity, SSIM and color reduction, the higher the image quality will be. Obviously, compared with the contrast algorithm, the Kblur of the restored image obtained by our algorithm decreased by $23.09 \%$, and the SSIM and Tonal reduction increased by $14.63 \%$ and $12.29 \%$ respectively.

Table 1 Evaluation of haze image parameters

\begin{tabular}{|c|c|c|c|c|}
\hline & Algorithm & Kblur & SSIM & $\begin{array}{c}\text { Tonal } \\
\text { reduction }\end{array}$ \\
\hline \multirow{4}{*}{1} & He. et al & 0.5214 & 0.7369 & 0.7213 \\
\cline { 2 - 5 } & Wang. et al & 0.5319 & 0.6698 & 0.6523 \\
\cline { 2 - 5 } & Our method & 0.4122 & 0.7690 & 0.7794 \\
\hline \multirow{4}{*}{2} & He. et al & 0.4432 & 0.8261 & 0.6312 \\
\cline { 2 - 5 } & Wang. et al & 0.5463 & 0.7530 & 0.6914 \\
\cline { 2 - 5 } & Our method & 0.4396 & 0.8869 & 0.7326 \\
\hline \multirow{4}{*}{3} & He. et al & 0.6124 & 0.6230 & 0.6626 \\
\cline { 2 - 5 } & Wang. et al & 0.6532 & 0.6021 & 0.6712 \\
\cline { 2 - 5 } & Our method & 0.5469 & 0.7123 & 0.6912 \\
\hline \multirow{3}{*}{4} & He. et al & 0.8861 & 0.6185 & 0.6872 \\
\cline { 2 - 5 } & Wang. et al & 1.2004 & 0.5171 & 0.6296 \\
\cline { 2 - 5 } & Our method & 0.7233 & 0.6965 & 0.7986 \\
\hline
\end{tabular}

\section{Conclusions}

Although the existing image-defogging algorithm can achieve a certain defogging effect, the restored images suffer from dark tones, low colour saturation, and colour distortion, which bring about extremes in image segmentation, target recognition, and target tracking. This paper proposes a method using dark channel prior and adaptive transmission rate. Compared with the traditional algorithm, the resolution of the reconstructed image, the structure similarity and the tone reduction degree are greatly improved, and the quality of the restored image can be significantly improved.

\section{References}

[1] Tan R T. Visibility in bad weather from a single image [C]. Proceedings of IEEE Conference on Computer Vision and Pattern Recognition, 2008, 1(1): 1-8.

[2] Dong Y Y, Bi D Y, He L Y et .al. Single Image Dehazing Algorithm Based On Non-Local Prior [J]. Acta Optica Sinica, 2017, 37(11): 1110001-1110010

[3] He K, Sun J, Tang X. Single Image Haze Removal Using Dark Channel Prior [J]. IEEE Transactions on Pattern Analysis \& Machine Intelligence, 2011, 33(12):2341-2353.

[4] Wang Y F, Yin C L, Huang Y M, et .al. Image haze removal using a bilateral filter [J]. Journal of Image and Graphics, 2014, 19(3):386-392.

[5] He K, Sun J, Tang X. Guided image filtering. [J]. IEEE Transactions on Pattern Analysis \& Machine Intelligence, 2013, 35(6):1397-1409.

[6] HUANG H, SONG J, WANG H F, et al. The Traffic Image Defogging Algorithm Based on Twice Filter in Haze Weather [J]. Science Technology and Engineering, 2016, 16(30):274-277.

[7] Li D P,Yu J, Xiao C B, et .al. No-reference quality assessment method for defogged images[J]. Journal of Image and Graphics, 2011, 16(9):1753-1757. 